EPJ Web of Conferences 66, 02050 (2014)

DOI: $10.1051 /$ epjconf/ 20146602050

(C) Owned by the authors, published by EDP Sciences, 2014

\title{
Shell model description of low-lying states in Po and Rn isotopes
}

\author{
Koji Higashiyama ${ }^{1, a}$ and Naotaka Yoshinaga ${ }^{2}$ \\ ${ }^{1}$ Department of Physics, Chiba Institute of Technology, Narashino, Chiba 275-0023, Japan \\ ${ }^{2}$ Department of Physics, Saitama University, Saitama City 338-8570, Japan
}

\begin{abstract}
Nuclear structure of the Po and Rn isotopes is theoretically studied in terms of the spherical shell model with the monopole- and quadrupole-pairing plus quadrupolequadrupole effective interaction. The experimental energy levels of low-lying states are well reproduced. The shell model results are examined in detail in a pair-truncated shell model. The analysis reveals the alignment of two protons in the $0 h_{9 / 2}$ orbital at spin 8 .
\end{abstract}

\section{Introduction}

Low-lying states in the light actinide region are successfully interpreted in terms of a few valence particles or holes with respect to the doubly magic nucleus ${ }^{208} \mathrm{~Pb}$. For example, the measured magnetic moments of the isomeric $8_{1}^{+}$states in ${ }^{206-214} \mathrm{Rn}$ were assigned to be the proton $\left(0 h_{9 / 2}\right)^{4}$ configurations to these states [1]. The low-lying near-yrast states were analyzed in terms of the interacting-bosonapproximation plus two quasi-particle model [2], where one of the bosons is replaced by a pair of nucleons coupled to angular momentum $J \geq 4$. A good agreement with experiment was achieved for both the energy spectra and electromagnetic transitions. Recently, many experimental investigations have been made not only for the low-lying states, but also for the high-spin states in this mass region [3-6]. For some nuclei, high-spin yrast isomers are interpreted as arising from neutron core excitations $[5,6]$.

In this paper, the band structures of the Po and $\mathrm{Rn}$ isotopes are studied in terms of the full-fledged shell model. Calculated energy levels are compared with the experimental data and wave functions are analyzed in terms of a pair truncated shell model.

\section{Shell Model Calculations}

For single-particle levels, all the six orbitals $0 h_{9 / 2}, 1 f_{7 / 2}, 0 i_{13 / 2}, 1 f_{5 / 2}, 2 p_{3 / 2}$, and $2 p_{1 / 2}$ are considered in the major shell of $82<N(Z)<126$ for neutrons (protons), and valence neutrons (protons) are treated as holes (particles). Adopted single-particle energies are extracted from experimental values of ${ }^{207} \mathrm{~Pb}$ [7] and ${ }^{209} \mathrm{Bi}$ [8], which are listed in Table 1.

The phenomenological monopole and quadrupole pairing plus quadrupole-quadrupole interaction is employed as an effective interaction. The Hamiltonian is explicitly written as

$$
H=\sum_{j m \tau} \varepsilon_{j \tau} c_{j m \tau}^{\dagger} c_{j m \tau}-\sum_{\tau}\left[G_{0 \tau} P_{\tau}^{\dagger(0)} P_{\tau}^{(0)}+G_{2 \tau} P_{\tau}^{\dagger(2)} \tilde{P}_{\tau}^{(2)}+\kappa_{\tau}: Q_{\tau} \cdot Q_{\tau}:\right]-\kappa_{v \pi} Q_{v} \cdot Q_{\pi},
$$

ae-mail: koji.higashiyama@it-chiba.ac.jp

This is an Open Access article distributed under the terms of the Creative Commons Attribution License 2.0, which permits unrestricted use, distribution, and reproduction in any medium, provided the original work is properly cited. 
Table 1. Adopted single-particle energies $\varepsilon_{j \tau}$ for neutron holes $(\tau=v)$ and proton particles $(\tau=\pi)$ in units of $\mathrm{MeV}$.

\begin{tabular}{ccccccc}
\hline$j$ & $2 p_{1 / 2}$ & $2 p_{3 / 2}$ & $1 f_{5 / 2}$ & $1 f_{7 / 2}$ & $0 h_{9 / 2}$ & $0 i_{13 / 2}$ \\
\hline Neutrons & 0.000 & 0.898 & 0.570 & 2.340 & 3.415 & 1.633 \\
Protons & 3.633 & 3.120 & 2.826 & 0.896 & 0.000 & 1.609 \\
\hline
\end{tabular}

where $c_{j m \tau}^{\dagger}$ is a nucleon creation operator, and $(j, m)$ represents a set of quantum numbers necessary to specify the state $(n, \ell, j, m)$. The interaction strengths among identical nucleons, $G_{0 \tau}, G_{2 \tau}$, and $\kappa_{\tau}$ are adjusted to fit the experimental data for singly closed nuclei, and those between neutrons and protons, $\kappa_{v \pi}$, are determined to reproduce the energy levels for open shell nuclei. The determined strengths of interactions are $G_{0 v}=0.130, G_{2 v}=0.010, \kappa_{v}=0.008, G_{0 \pi}=0.110, G_{2 \pi}=0.000, \kappa_{\pi}=0.038$, and $\kappa_{\gamma \pi}=-0.040\left(G_{0 \tau}\right.$ in units of $\mathrm{MeV}$, and $G_{2 \tau}$ and $\kappa_{\tau}$ both in units of $\left.\mathrm{MeV} / b^{4}\right)$. It should be noted that the interaction strengths adopted in the present calculations are assumed to be the same for all the nuclei. The calculations are performed using the computer code MS [9].

In Figs. 1 and 2, the measured spectra for even-even Rn and Po isotopes are compared with the shell model results. Experimentally, the level spacings between the adjacent yrast states decrease monotonically as spin $I$ increases up to spin 8 . The $8_{1}^{+}$isomers with half-lives of 200-700 ns have been identified in these isotopes. [In the case of ${ }^{210} \mathrm{Rn}$, the relative position of the $8_{1}^{+}$state and the ground $\left(0_{1}^{+}\right)$state remains unknown.] For the nuclei considered, the even-spin yrast sequences are well reproduced except for the $8_{1}^{+}$states, which are lower in energy than the experimental data. For a better reproduction of the $8_{1}^{+}$states, multipole pairing interactions more than quadrupole might be necessary. Concerning other states, good agreements between theory and experiment are achieved.

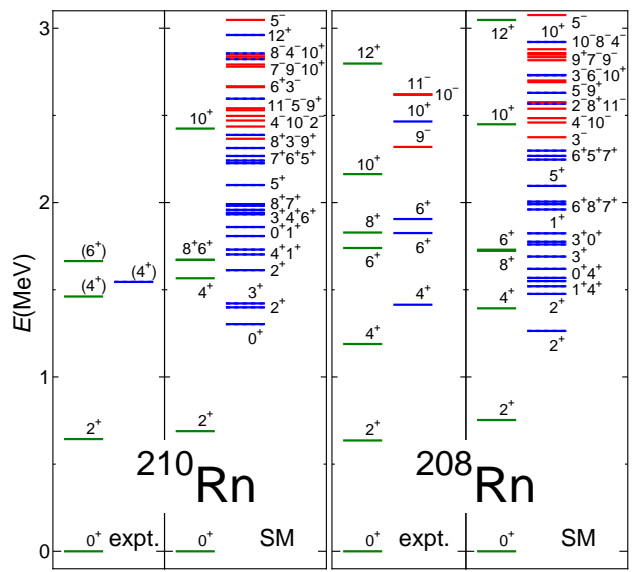

Figure 1. Comparison of the experimental energy levels with those of the shell model for ${ }^{208} \mathrm{Rn}$ and ${ }^{210} \mathrm{Rn}$. The experimental data are taken from Refs. [12, 13].

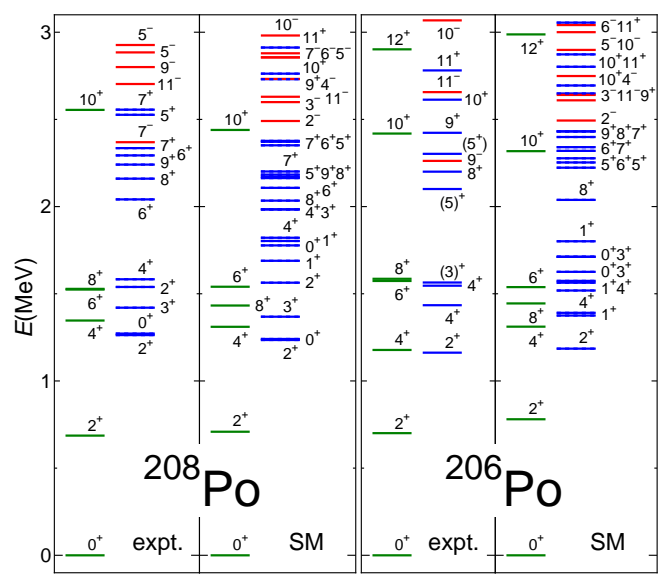

Figure 2. Same as figure 1 , but for ${ }^{206} \mathrm{Po}$ and ${ }^{208} \mathrm{Po}$. The experimental data are taken from Refs. [13, 14]. 


\section{Analysis of wave functions in terms of PTSM}

In order to investigate collective behavior at low energies and the effect of the single particle excitations above spin 8, the energy spectra in the shell model are compared with those in a pair-truncated shell model (PTSM) [10, 11]. In the present scheme, the building blocks of the PTSM are mainly the angular momenta zero $(S)$ and two $(D)$ collective pairs, and non-collective $H$ pairs. The $S$ and $D$ pair-creation operators are defined as

$$
\begin{aligned}
S^{\dagger} & =\sum_{j} \alpha_{j} A_{0}^{\dagger(0)}(j j), \\
D_{M}^{\dagger} & =\sum_{j_{1} j_{2}} \beta_{j_{1} j_{2}} A_{M}^{\dagger(2)}\left(j_{1} j_{2}\right),
\end{aligned}
$$

where the pair creation operator of two nucleons in the orbitals $j_{1}$ and $j_{2}$ with total angular momentum $J$ and its projection $M$ is defined as

$$
A_{M \tau}^{\dagger(J)}\left(j_{1} j_{2}\right)=\sum_{m_{1} m_{2}}\left(j_{1} m_{1} j_{2} m_{2} \mid J M\right) c_{j_{1} m_{1}}^{\dagger} c_{j_{2} m_{2}}^{\dagger}=\left[c_{j_{1} \tau}^{\dagger} c_{j_{2} \tau}^{\dagger}\right]_{M}^{(J)}
$$

In the present approach, the structure coefficients $\alpha$, and $\beta$ are determined by variation.

The $H$ pair creation operators for neutrons are defined as

$$
\begin{aligned}
H_{1 v M}^{(K) \dagger} & =\left[c_{v 5 / 2}^{\dagger} c_{v 5 / 2}^{\dagger}\right]_{M}^{(K)}, \quad \text { with } \quad K=0,2,4, \\
H_{2 v M}^{(K) \dagger} & =\left[c_{v 5 / 2}^{\dagger} c_{v 1 / 2}^{\dagger}\right]_{M}^{(K)}, \quad \text { with } \quad K=2,3, \\
H_{3 v M}^{(K) \dagger} & =\left[c_{v 5 / 2}^{\dagger} c_{v 3 / 2}^{\dagger}\right]_{M}^{(K)}, \quad \text { with } \quad K=1,2,3,4, \\
H_{4 v M}^{(K) \dagger} & =\left[c_{v 13 / 2}^{\dagger} c_{v 13 / 2}^{\dagger}\right]_{M}^{(K)}, \quad \text { with } \quad K=0,2,4,6,8,10,12,
\end{aligned}
$$

and those for protons are defined as

$$
\begin{aligned}
H_{1 \pi M}^{(K) \dagger} & =\left[c_{\pi 9 / 2}^{\dagger} c_{\pi 9 / 2}^{\dagger}\right]_{M}^{(K)}, \quad \text { with } \quad K=0,2,4,6,8, \\
H_{2 \pi M}^{(K) \dagger} & =\left[c_{\pi 13 / 2}^{\dagger} c_{\pi 13 / 2}^{\dagger}\right]_{M}^{(K)}, \quad \text { with } \quad K=0,2,4,6,8,10,12 .
\end{aligned}
$$

Using the $S, D$, and $H$ pair-creation operators, a many-body wave function of like nucleons can be constructed as

$$
\left|\Psi_{n_{s}, n_{d}, n_{h}}(I \eta)\right\rangle=\left(S^{\dagger}\right)^{n_{s}}\left(D^{\dagger}\right)^{n_{d}}\left(H_{i}^{\dagger}\right)^{n_{h}}|-\rangle .
$$

The Hamiltonian for this truncated space (PTSM space) is set identical to that used in the shell model.

In Fig. 3, the energy levels obtained by the PTSM for ${ }^{208} \mathrm{Rn}$ are compared with those of the shell model. There is a good correspondence between the energy levels of the shell model and those in the PTSM. This means that the model space spanned by the PTSM is adequate enough for describing the shell model results. In search for the microscopic structure of the yrast band, the wave functions obtained by the PTSM are analyzed. In Fig. 4, the expectation values of the numbers of pairs in the PTSM wave functions for ${ }^{208} \mathrm{Rn}$ isotopes are shown as a function of spin $I$. It is found that the valence neutron excitation is responsible for the low-lying states, while the effect of the alignment of two $0 h_{9 / 2}$ protons becomes apparent above spin 8 . Furthermore, the alignment of two $0 i_{13 / 2}$ neutrons plays a decisive role in describing the states above spin 14. 


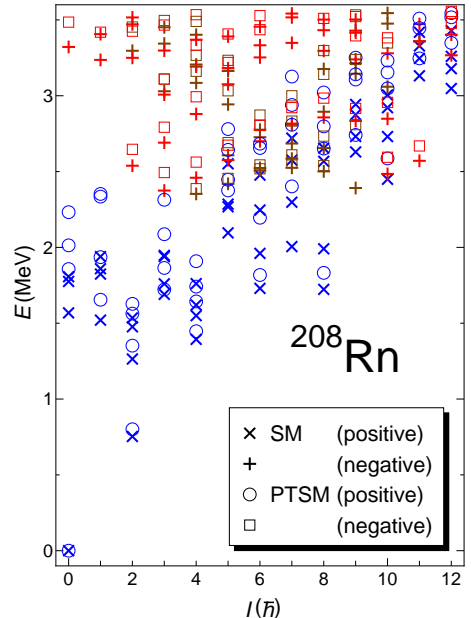

Figure 3. Comparison of the calculated energy levels in the PTSM and the shell model results for ${ }^{208} \mathrm{Rn}$.

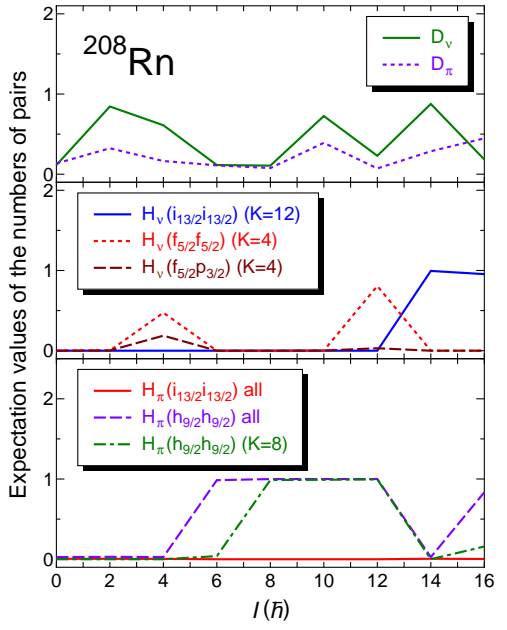

Figure 4. The expectation values of the numbers of $D$ and $H$ pairs calculated in the PTSM.

\section{Summary}

Full-fledged shell model calculations are performed for the even-even nuclei in the light actinide region. The monopole and quadrupole pairing plus quadrupole-quadrupole interaction is employed as an effective interaction. Our calculation reproduces quite well the energy levels for the nuclei considered. In order to interpret the shell model results, we compare those with the PTSM calculations. From the results of the expectation values of the numbers of pairs, it is found that the valence neutron excitation plays an essential role in describing the low-lying states, and the pair of $0 h_{9 / 2}$ protons is indispensable for the states above spin 8 .

\section{References}

[1] K. H. Maier et al., Hyperfine Interact. 9, 87 (1981).

[2] A. Zemel and J. Dobes, Phys. Rev. C 27, 2311 (1983).

[3] G. D. Dracoulis et al., Phys. Rev. C 72, 064319 (2005).

[4] A. M. Baxter et al., Phys. Rev. C 71, 054302 (2005).

[5] G. D. Dracoulis et al., Phys. Rev. C 77, 034308 (2008).

[6] G. D. Dracoulis et al., Phys. Rev. C 80, 054320 (2009).

[7] F. G. Kondev and S. Lalkovski, Nucl. Data Sheets 112, 707 (2011).

[8] M. J. Martin, Nucl. Data Sheets 63, 723 (1991).

[9] N. Yoshinaga, code MS, unpublished.

[10] N. Yoshinaga and K. Higashiyama, Phys. Rev. C 69, 054309 (2004).

[11] K. Higashiyama and N. Yoshinaga, Phys. Rev. C 83, 034321 (2011).

[12] E. Browne, Nucl. Data Sheets 99, 483 (2003).

[13] M. J. Martin, Nucl. Data Sheets 108, 1583 (2007).

[14] F. G. Kondev, Nucl. Data Sheets 109, 1527 (2008). 